\title{
Development and mechanical performance of a new kind of bridge seismic isolator for low seismic regions
}

\author{
H. Zhang, J. Li and T. Peng* \\ Department of Bridge Engineering, Tongji University, Shanghai, China
}

Received 24 September 2011

Revised 3 December 2012

Accepted 29 January 2013

\begin{abstract}
The concept of fibre-reinforced plate elastomeric isolator (FRPEI) is introduced firstly in this paper. Three FRPEI specimens have been constructed to evaluate the mechanical performance of the isolators by performing vertical and horizontal tests. The research focuses on the compression stiffness, the shear stiffness, the hysteretic characteristic and the vertical bearing capacity of the isolators. The experimental results show that the mechanical performance of FRPEIs can meet the requirements of bridge rubber bearings and the energy dissipation capacity is better than that of general laminated rubber bearings. Therefore, it is feasible to use FRPEIs in seismic isolation of short span bridges in low seismic regions. Theoretical and finite element methods have also been employed and the deformation assumptions applied in the theoretical method are also verified by FEM. By comparing the differences of the results of different methods, the effectivenesses of the theoretical and finite element methods are evaluated and some considerations on isolator design are proposed.
\end{abstract}

Keywords: Glass fibre, finite element analysis (FEA), mechanical testing, bridge seismic isolator

\section{Introduction}

Bridge seismic isolation, which means to protect bridge structures by isolating and/or dissipating earthquake energy with isolation devices such as isolator and damper, is a practical and valuable technique for seismic design. The bridge structures are not required to have high strength to resist the earthquake loads and can remain elastic under earthquake if designed properly. Therefore, bridge seismic isolation has been widely used in recent years.

The existing bridge seismic isolators are mostly expensive, massive and heavy [5], for example some bearings combined with steel dampers or viscous dampers are capable of providing better energy dissipation, vertical load bearing capacity and horizontal displacement capacity, but their applications are restricted by the complex configuration and large size [1,2], particularly in many developing countries and economically undeveloped areas, where even some important bridges cannot apply this ideal technique. For some short span bridges in low seismic regions, large-sized bridge seismic isolators are also rejected and several kinds of elastomeric isolators are preferred for economic reasons.

The existing elastomeric isolators include lead rubber bearings, high damping rubber bearings and common laminated rubber bearings. Lead rubber bearings [4] and high damping rubber bearings [3] are widely used in seismic

*Corresponding author: T. Peng, Department of Bridge Engineering, Tongji University, Shanghai 200092, China. Tel.: +86 131 6268 3210; E-mail: ptb@tongji.edu.cn. 
design of bridges, where they exhibit satisfactory energy dissipation capacities. General laminated rubber bearings are made of general rubber and relatively economical but have little energy dissipation capacity. A more economical and lighter seismic isolator, named fibre-reinforced elastomeric isolator (FREI) was developed by Kelly [5], which is suitable for seismic isolation of short span bridges in low seismic regions. He pointed out that the weight and cost of the isolator could be reduced by replacing the steel reinforcement in laminated rubber bearing with fibre reinforcement. The weight is reduced because that the fibre material with an elastic stiffness of the same order as steel has a relatively low density; the cost savings may be realized since the use of fibre allows a simpler, less laborintensive manufacturing process. The theoretical equations of compression stiffness and bending stiffness with the consideration of the flexibility of the reinforcement were derived [5]. Meanwhile, experimental studies had been conducted and the results showed that FREIs had almost the same compression stiffness and bending stiffness as the steel reinforced isolators, but FREIs could provide greater energy dissipation capacity.

Since the promotion of the concept of FREI, many researchers from different countries have made their efforts to study and improve the performance of FREI. Currently, there are three methods to study the mechanical performance of FREI, including theoretical method, experimental method and finite element method (FEM). The theoretical researches were mainly carried out by Kelly [5-7] and Tsai [8-12] etc. In these analyses, the rubber in the isolator was assumed to be strictly incompressible. The following two assumptions were made when they conducted compression analysis of FREI: 1) horizontal planes remain planar and 2) points on a vertical line lie on a parabola after loading. Compression stiffness and bending stiffness of infinitely long strip [7], rectangular [9] and circular [10] FREI were widely studied. Tension flexibility of the reinforcement was taken into account in these analyses. The equations of the compression stiffness and the bending stiffness were derived for FREI of different shapes. Parametric studies showed that the compression stiffness and the bending stiffness of FREI were affected by the shape factor of the isolator and the flexibility of the reinforcement.

Kelly has also conducted some experimental researches on both circular and strip FREI. The mechanical performance of circular FREI with Kevlar fibre reinforcement was studied by Kelly [6]. The results indicated that the flexibility of the fibre reinforcement had only a small effect on the shear stiffness of the isolator. The stiffness was reduced to around $80-85 \%$ of that of the steel-reinforced isolator with the same size and thickness of the elastomer. Another finding of the tests was the improvement of the energy dissipation of the bearing with fibre reinforcement. Kelly [6] pointed out that a possible explanation for this effect was the slip and friction effect between fibres. Since each individual fibre of a single plane of reinforcement was made up of an extremely large number of single fibres twisted into a thread, relative slip between fibres may happen in the tests. Similar experiments were conducted to strip isolators with carbon fibre in 2002 [7]. In addition, some relevant experimental researches were conducted by Moon [13,14], Kang [15], Mordini [16] and Ashkezari [17]. In these tests, different types of fibres were considered, including carbon fibre, glass fibre, Kevlar fibre etc., and the results of the tests showed that carbon fibre was the best one, and although the glass fibre appeared to be of very low quality and low-cost, it had sufficient stiffness and strength for the requirement of isolators.

Compared with the large amount of theoretical and experimental researches, FEM researches are relatively fewer. By taking the fibre reinforcement as orthotropic material and modeling the rubber with Ogden model, Mordini [16] conducted some parametric studies. The influences of vertical displacement on vertical stiffness and horizontal stiffness were studied and the equations of the vertical and horizontal stiffness were derived by taking them as the functions of the vertical and horizontal displacements and fitting the results with second order polynomial surfaces.

Most of the researches in the past were based on the fibre fabric which is relatively rigid in tension and relatively flexible in bending. The fibre fabric was made up of many individual fibres grouped in strands and coiled into a cord of sub millimeter diameter. In this research, a new type of fibre reinforcement named Fibre reinforced plates, which have great stiffness both in tension and bending, is promoted. Fibre reinforced plates are made from fibre fabric and epoxy resin and used to replace the steel plates in the laminated rubber bearing to develop a new isolator named fibre-reinforced plate elastomeric isolator (FRPEI). Three specimens were manufactured to study the mechanical performance of this new kind of isolator by conducting vertical and horizontal tests. 


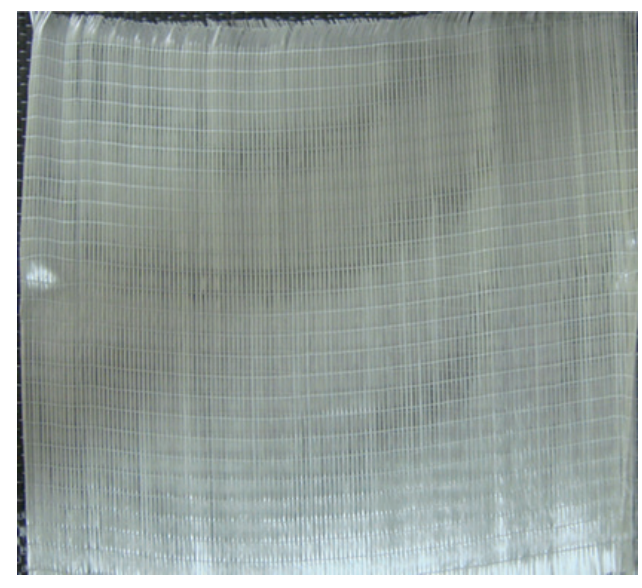

Fig. 1. Fiber fabric.

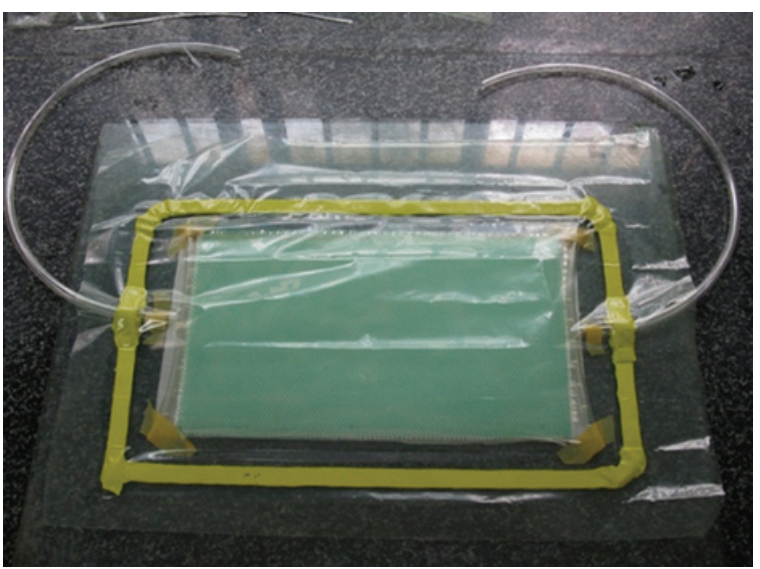

Fig. 3. Lay pipes and vacuum bag.

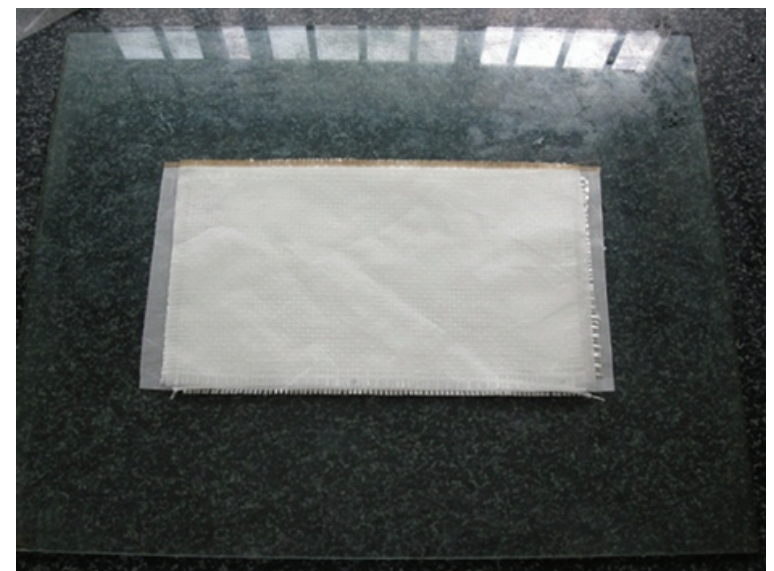

Fig. 2. Lay down glass fiber fabric and release fabric.

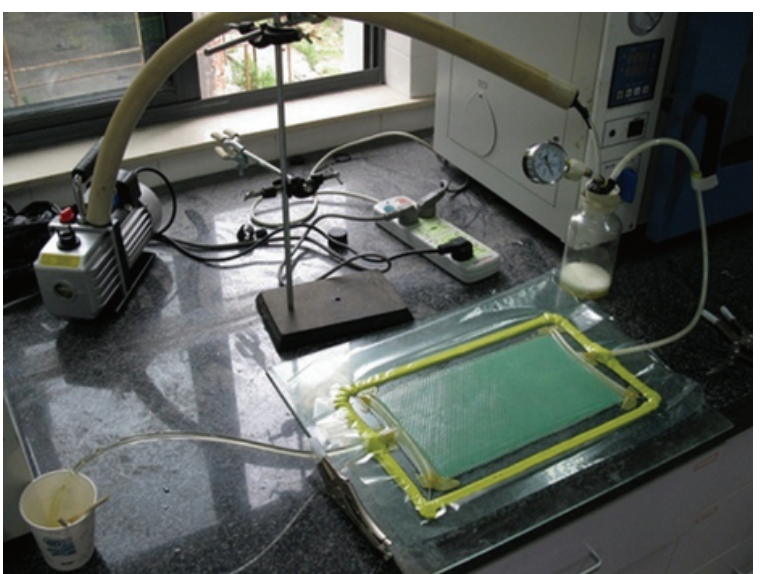

Fig. 4. Pump the formulated resin.

\section{Description of the specimens and tests}

\subsection{Description of the isolator specimens}

The fibre reinforcement used in FREIs is relatively rigid in tension and relatively flexible in bending, so the flexibility of the reinforcement may result in the reduction of the vertical, shear and bending stiffness. Therefore, the fibre reinforced plates with great stiffness both in tension and bending may be an alternative for the reinforcement. Fibre reinforced plates are made from fibre fabric and epoxy resin. The fibre fabric, as shown in Fig. 1, has large stiffness and strength along the fibres but small stiffness and strength perpendicular to the fibres. Steps to manufacture the fibre reinforced plates are as follows:

a) Apply release agent twice on the glass plate where fibre fabrics will be put on, the time interval is 10 minutes.

b) Put a piece of release fabric on the glass plate, which can make sure a successful release of the completed reinforcement.

c) Lay down the fibre fabrics by specified stacking sequence on the release fabric, as shown in Fig. 2.

d) Put another release fabric on the fibre fabrics and lay diversion net.

e) Set diversion pipe, resin inlet pipe and air outlet pipe beside the pre-molded fibre fabrics, as shown in Fig. 3.

f) Connect the vacuum pump with the air outlet pipe; put the resin inlet pipe in the resin container, as shown in Fig. 4. After formulated, the resin should have a standing time to eliminate air bubbles. 


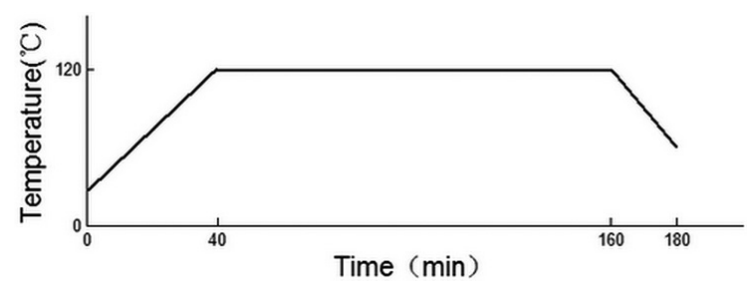

Fig. 5. Time-temperature curve.

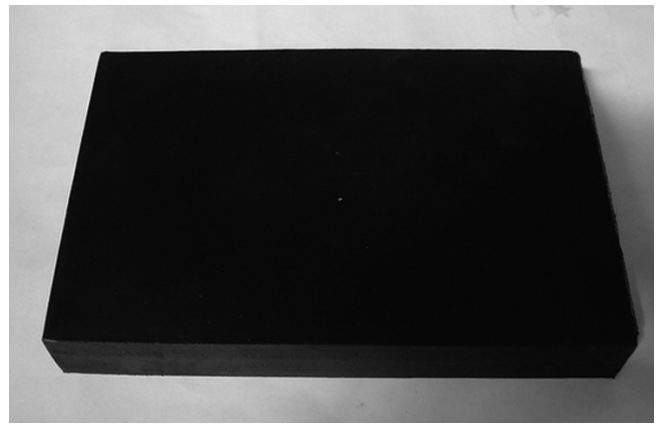

Fig. 6. An FRPEI specimen.

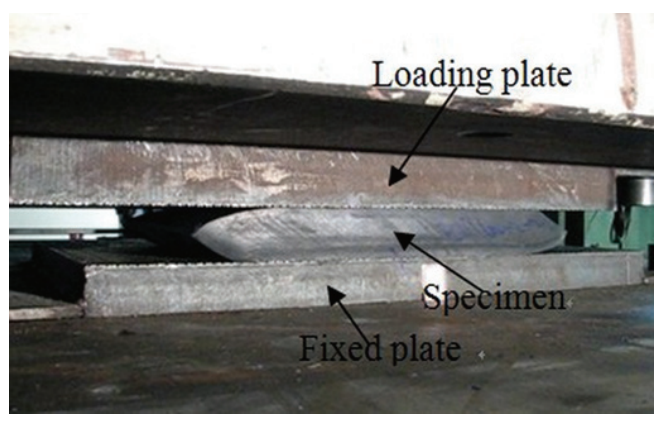

Fig. 7. Overall view of the test.

g) Finally, put the product in the electric drying oven, and heat it to $120^{\circ} \mathrm{C}$ for two hours, the time-temperature curve applied is presented in Fig. 5.

The plates can have different thicknesses and mechanical performance, since they can consist of different layers of fibre fabrics with different ply orientations. The ply orientations are of $\left[0^{\circ} / 90^{\circ} / 0^{\circ} / 90^{\circ} / 0^{\circ} / 90^{\circ}\right]$ and $\left[0^{\circ} / 90^{\circ} /\right.$ $0^{\circ} / 90^{\circ} / 0^{\circ} / 90^{\circ} / 0^{\circ} / 90^{\circ} / 0^{\circ} / 90^{\circ}$ ] for six and ten layers respectively, which are used in the specimens in this research. The glass fibre is adopted to make fibre reinforced plates in this research.

Three FRPEI specimens were designed and manufactured. The detail dimensions are tabulated in Table 1 and one of the FRPEI specimens is presented in Fig. 6.

\subsection{Description of the tests}

All experiments are conducted with a $20 \mathrm{MN}$ compression-shear combined testing machine. As shown in Fig. 7 , the testing system consists of a loading plate and a fixed plate. Vertical loading and horizontal cyclic loading can be applied by the loading plate on the top of the specimen and the fixed plate kept fixed at the bottom of the specimen.

Three series of tests are conducted to study the mechanical performance of FRPEIs, including vertical compression stiffness test, shear stiffness and hysteretic characteristic test and vertical bearing capacity test. In the vertical compression stiffness test, the vertical load is imposed monotonically on the isolator from $0 \mathrm{MPa}$ to $10 \mathrm{MPa}$ (service pressure of the isolators) to test the nominal compression stiffness of the specimens. In the shear stiffness and hysteretic characteristic test, the horizontal loading applied to each specimen is shown on Fig. 8. It is a displacement control mode test as seen in the figure. To make the maximum shear strain of each specimen achieve $100 \%$, the specimen with greater thickness has greater displacement amplitude. Taking No.1 specimen for example, since total thickness of rubber in the specimen is $26.9 \mathrm{~mm}$, the peak of the cyclic displacement is about $26 \mathrm{~mm}$. The vertical pressure of the specimens is kept to be $10 \mathrm{MPa}$ for all horizontal tests. There is no bonding between the specimens and the test plates. The shear load is transferred from the loading plate to the specimens and then to the fixed plate by the occlusion and friction force between the plates and the specimens as seen in Fig. 7. In the vertical bearing capacity test, the vertical load is increased monotonically on the isolator from $10 \mathrm{MPa}$ until the specimens reach the ultimate bearing capacity. 


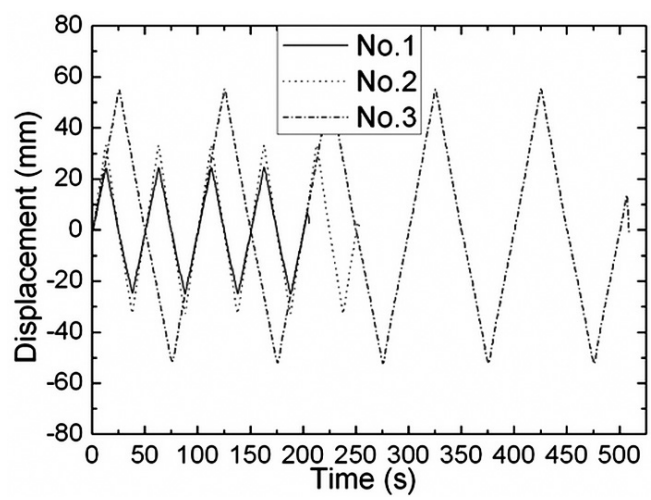

Fig. 8. Horizontal cyclic loading applied to each specimen.

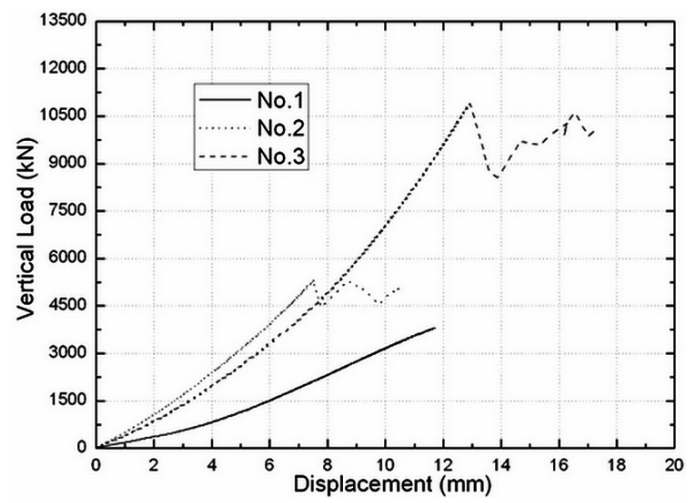

Fig. 9. Vertical tests of FRPEI specimens.

\section{Analysis of the tests results}

\subsection{Vertical tests}

Vertical tests are conducted to study the bearing capacity and compression modulus of the FRPEI specimens. The compression modulus can be derived from the following equation:

$$
E_{c}=\frac{\sigma_{10}-\sigma_{4}}{\varepsilon_{10}-\varepsilon_{4}}
$$

where $E_{c}$ is the compression modulus, $\sigma_{10}$ and $\sigma_{4}$ are the compression stresses of $10 \mathrm{MPa}$ and $4 \mathrm{MPa}$ respectively, $\varepsilon_{10}$ and $\varepsilon_{4}$ are the corresponding strains.

The results of the three specimens' vertical tests are presented on Fig. 9, from which the vertical bearing capacities of the specimens are $3796 \mathrm{kN}, 5318 \mathrm{kN}$ and $10891 \mathrm{kN}$, respectively. By dividing the load by the area of the specimens, the ultimate pressure of each specimen can be obtained, which are $63 \mathrm{MPa}, 51 \mathrm{MPa}$ and $58 \mathrm{MPa}$ for the No. 1, No. 2 and No. 3 specimen, respectively. According to the rubber bearing codes (JT/T4-2004), the ultimate pressure of laminated rubber bearing needs to be $70 \mathrm{MPa}$. It can be learnt that FRPEIs have a little smaller bearing capacity. However, since the service pressure of laminated rubber bearing is about $10 \mathrm{MPa}$, it can be observed that the vertical bearing capacity can still meet the requirement of rubber bearing with large safety margin. With Eq. (1), the compression moduli of the specimens are calculated to be $439 \mathrm{MPa}, 493 \mathrm{MPa}$ and $507 \mathrm{MPa}$ for No. 1, No. 2 and No. 3 specimen, respectively.

\subsection{Horizontal tests}

The shear modulus of the specimens is tested according to the rubber bearing codes (JT/T4-2004). It can be calculated as follows:

$$
G=\frac{\tau_{1}-\tau_{0.3}}{\gamma_{1}-\gamma_{0.3}}
$$

where $G$ is the shear modulus, $\tau_{1}$ and $\tau_{0.3}$ are the shear stresses of $1 \mathrm{MPa}$ and $0.3 \mathrm{MPa}$ respectively, $\gamma_{1}$ and $\gamma_{0.3}$ are the corresponding shear strains. The shear moduli obtained from the tests for specimen No. 1, No. 2 and No. 3 are $0.810 \mathrm{MPa}, 0.800 \mathrm{MPa}$ and $0.815 \mathrm{MPa}$ respectively. Material tests have been conducted and the shear modulus of the rubber is about $1 \mathrm{MPa}$ in the test specimens. Reduction in shear modulus is found in all three specimens, which may possibly be caused by the shear strain and warping deformation of the FRP reinforcement. The effective damping ratio of a bearing is a useful parameter to evaluate the energy dissipation capacity of the bearing and horizontal 


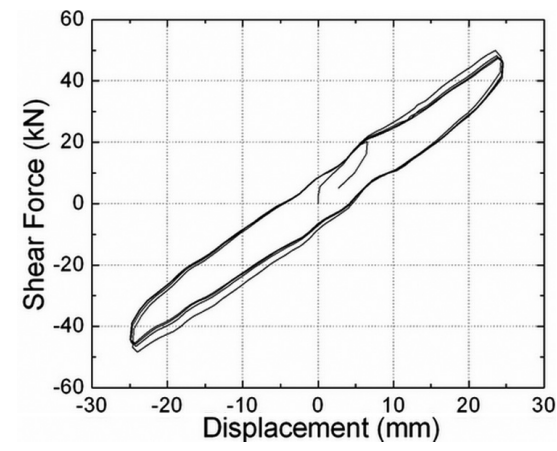

(a) Specimen No. 1

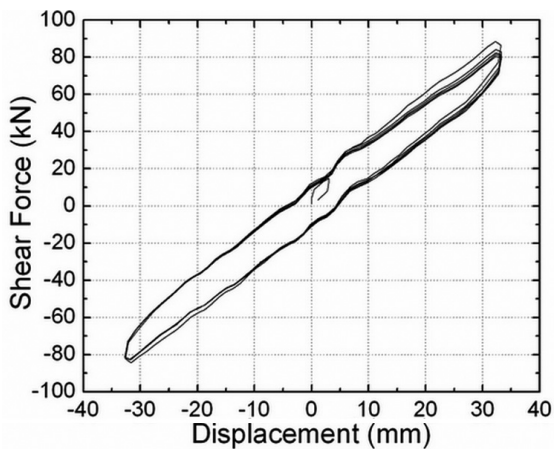

(b) Specimen No. 2

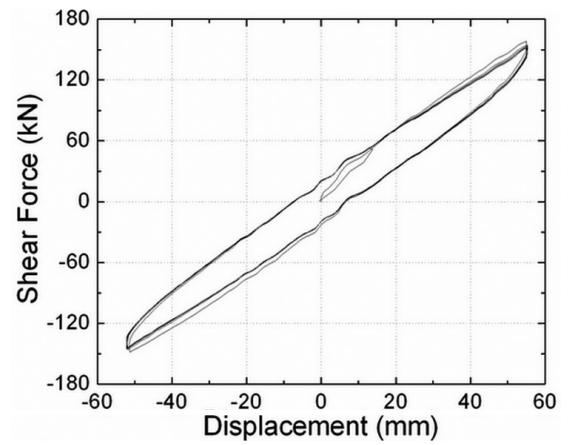

(c) Specimen No. 3

Fig. 10. Hysteretic curves of the specimens.

cyclic loading tests are conducted to obtain the effective damping ratios of the specimens. The damping ratio can be calculated from the hysteretic curves of the specimens by the following equation [19]:

$$
\xi_{e q}=\frac{A_{h}}{2 \pi V_{m} \Delta_{m}}
$$

where $A_{h}$ is the area of a hysteretic curve cycle, $V_{m}=1 / 2\left(\left|V_{\max }\right|+\left|V_{\min }\right|\right), \Delta_{m}=1 / 2\left(\left|\Delta_{\max }\right|+\left|\Delta_{\min }\right|\right), V_{\max }$ and $V_{\min }$ are the largest and smallest shear forces, $\Delta_{\max }$ and $\Delta_{\min }$ are the corresponding displacements. The equation indicates that for the same cyclic load, the larger the area of the hysteretic curve cycle, the greater the energy dissipation capacity.

The hysteretic curves of the three specimens are shown in Fig. 10. With Eq. (3), the effective damping ratios for the specimens were derived, which are $9.4 \%, 7.2 \%$ and $7.1 \%$ for No. 1, No. 2 and No. 3 specimens respectively. Compared with the results shown in a reference [5], the damping ratio is a little smaller. A possible reason may be that the fibre reinforcement used in this paper, which was bonded by resin, has great stiffness in tension and bending. The interfacial slip between fibres may be prevented, so the frictional damping is reduced. However, compared with laminated rubber bearing, FRPEI has larger energy dissipation capacity.

\section{Simulation of the tests with finite element method}

Finite element method can be employed to study the compression and shear stiffness of FRPEI. Moreover, an insight into the stress distribution of rubber and FRP reinforcement can be achieved. There are two types of materials in FRPEIs: fibre reinforcement and rubber. It is critical to model the mechanical performance of the materials correctly. Furthermore, simulation of the load type in the tests is essential. MSC.Marc is applied to carry out FEM analysis. 


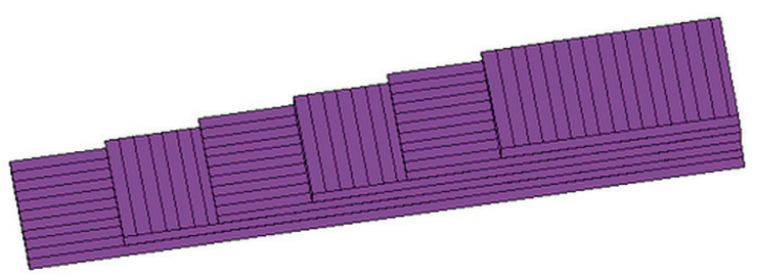

Fig. 11. The composite model in MSC.Marc.

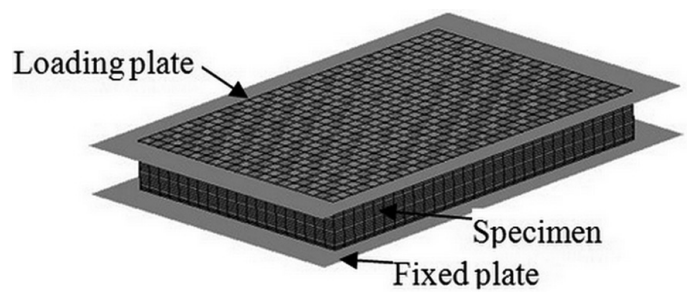

Fig. 12. Finite element model of the experiment.

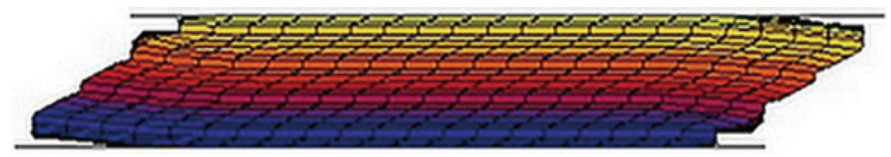

Fig. 13. Finite element model with 200 percent shear strain.

Rubber is a typical nonlinear material. It can have large strain with small stress and the largest tensile strain can get around 500\% 1000\%. Thus, it may be unreasonable to model rubber with only modulus and Poisson ratio. MSC.Marc provides a material model named Mooney-Rivlin to simulate rubber. The expression of two-parameter Mooney-Rivlin model is given in Eq. (4) below:

$$
W=C_{10}\left(I_{1}-3\right)+C_{01}\left(I_{2}-3\right)
$$

Where $W$ is the generalized strain energy function, $C_{10}$ and $C_{01}$ are the Rivlin factors, and

$$
\begin{aligned}
& I_{1}=\lambda_{1}^{2}+\lambda_{2}^{2}+\lambda_{3}^{2} \\
& I_{2}=\lambda_{1}^{2} \lambda_{2}^{2}+\lambda_{2}^{2} \lambda_{3}^{2}+\lambda_{3}^{2} \lambda_{1}^{2}
\end{aligned}
$$

which are the first and second Green strain invariant, where $\lambda_{1}, \lambda_{2}$ and $\lambda_{3}$ are the tensile rates of the three directions respectively. Rubbers with different hardness have different $C_{10}$ and $C_{01}$ factors. Tensile and shear material tests have been conducted to determine the factors of the rubber used in the tested specimens. The factors are as follows:

$$
C_{10}=0.433 \mathrm{MPa} \quad C_{01}=0.146 \mathrm{MPa}
$$

Fibre reinforcement is a composite material with anisotropic mechanic properties. MSC.Marc provides composite model to simulate composite materials. The model is composed of different layers. The material, thickness and angle of the layers can be set different. In this paper, the material of the layers is uniform and the included angle of the adjacent layers is $90^{\circ}$. The factors of the material are as follows:

$$
E_{11}=38.6 \mathrm{GPa} E_{22}=E_{33}=8.27 \mathrm{GPa} \nu_{12}=\nu_{23}=\nu_{31}=0.3 \quad G_{12}=G_{23}=G_{31}=4.14 \mathrm{GPa}
$$

where 1 represents the direction along the fibre, 2 represents the direction perpendicular to the fibre in the fabric plane, and 3 represents the direction perpendicular to the fibre fabric. The ply orientations are of $\left[0^{\circ} / 90^{\circ} / 0^{\circ} / 90^{\circ} / 0^{\circ} / 90^{\circ}\right] \mathrm{s}$ for the reinforcement of specimens No. 1 and No.2, and $\left[0^{\circ} / 90^{\circ} / 0^{\circ} / 90^{\circ} / 0^{\circ} / 90^{\circ} / 0^{\circ} / 90^{\circ} / 0^{\circ} /\right.$ $90^{\circ}$ ] for specimen No.3. Figure 11 shows the composite model in MSC.Marc.

The specimen is modelled as a deformable contact body. The loading plate and fixed plate are modelled as rigid plates, as shown in Fig. 12. There are no relative displacement between the interface of the test plates and specimen, and detachment happens when tension force appears. The finite element model of the test is shown in Fig. 12 and the deformed model with a deformation of $200 \%$ shear strain is presented in Fig. 13. As observed in the experiments, the separation between the specimens and the test plates occurred because of the rolling over effect caused by large lateral displacement. 


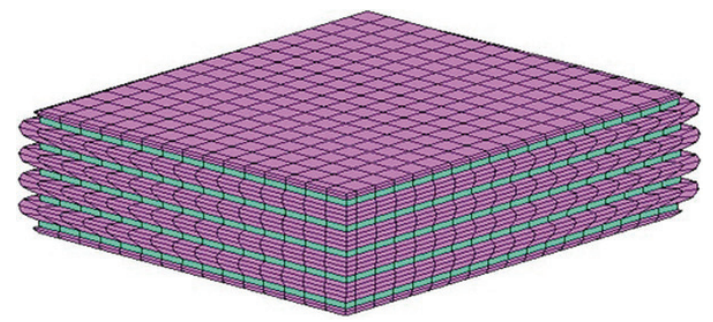

(a) View from the symmetry axis

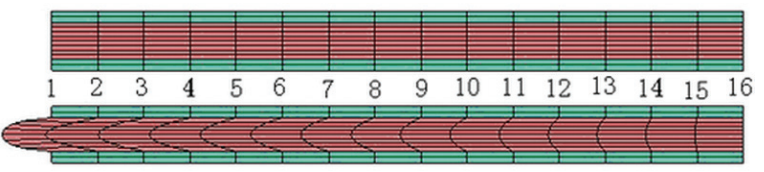

(b) Comparison before and after loading

Fig. 14. Deformation condition of an FRPEI under pressure.

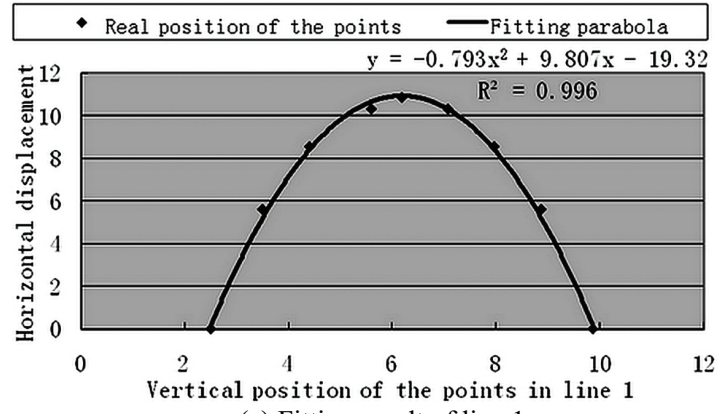

(a) Fitting result of line 1

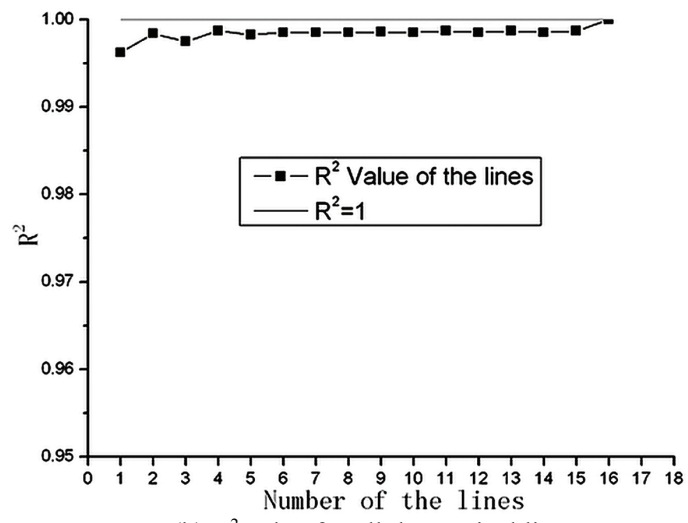

(b) $\mathrm{R}^{2}$ value for all the vertical lines

Fig. 15. Fitting of the vertical lines.

\subsection{Deformation analysis of FRPEIs}

Theoretical analysis of FREI's compression stiffness is based on the deformation assumptions that horizontal planes remain horizontal and points on a vertical line lie on a parabola after vertical loading and these assumptions can be verified by FEM. The deformation condition of an FRPEI (one rubber layer and two reinforcements) under $70 \mathrm{MPa}$ pressure is shown in Fig. 14. To verify the assumption that points on a vertical line lie on a parabola after vertical loading, the vertical lines are numbered from left to right as shown in the figure. By fitting the deformed lines with quadratic polynomial, the difference between the deformed vertical line and a parabola can be studied. The fitting result of line 1 is shown in Fig. 15(a). $R^{2}$ is the precision index of the fitting. It is defined by the following equation.

$$
R^{2}=1-\frac{\sum_{i=1}^{n}\left(y_{i}-\hat{y}_{i}\right)^{2}}{\sum_{i=1}^{n}\left(y_{i}-\bar{y}\right)^{2}}
$$

where $\hat{y}_{i}$ is the predictive value arrived from the regression function; $\bar{y}$ is average value of $y_{i}$. Greater precision is arrived when $R^{2}$ is closer to 1 . It can be learnt from Fig. 15(a) that line 1 is nearly a parabola after deformed by vertical loading. The fitting values of $R^{2}$ for all the numbered vertical lines are shown in Fig. 15(b). The values of $R^{2}$ for all the lines are greater than 0.99 , which indicates that the assumption about the vertical lines in theoretical analysis is reasonable. There is no deformation for line 16 (the center vertical line of the specimen). It can be taken as a parabola with zero coefficients and the $R^{2}$ is equal to 1 . 


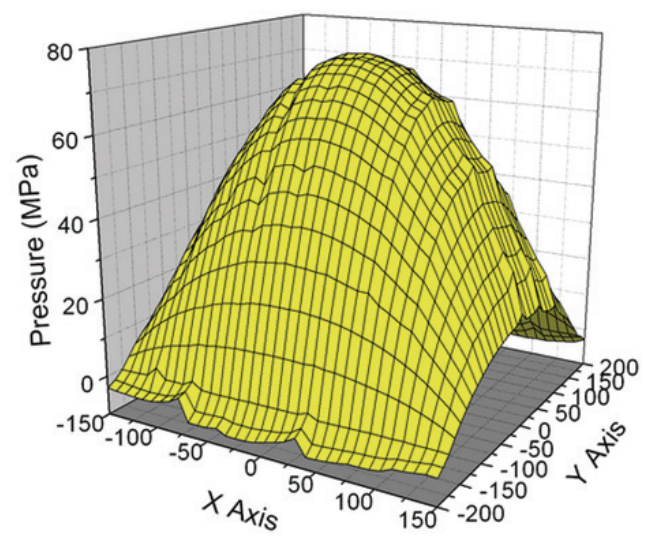

(a) Stress distribution of an FEM analysis

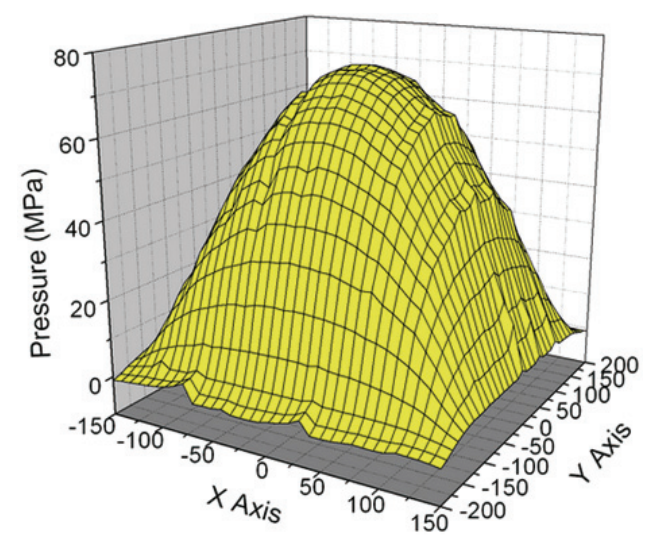

(b) Stress distribution result of an theoretical analysis

Fig. 16. Stress distribution in rubber of specimen No. 2.

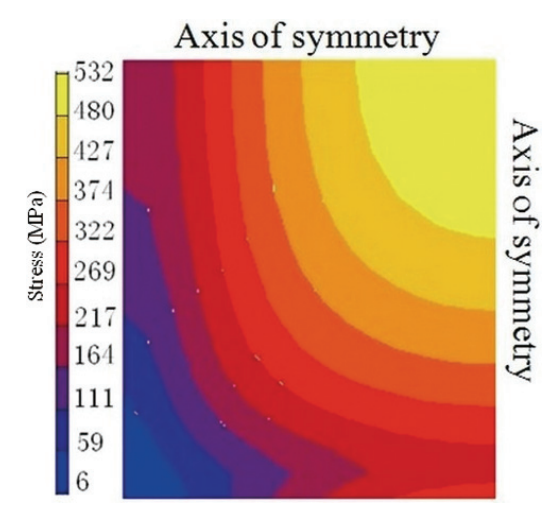

Fig. 17. Von Mises stress distribution in FRP reinforcement.

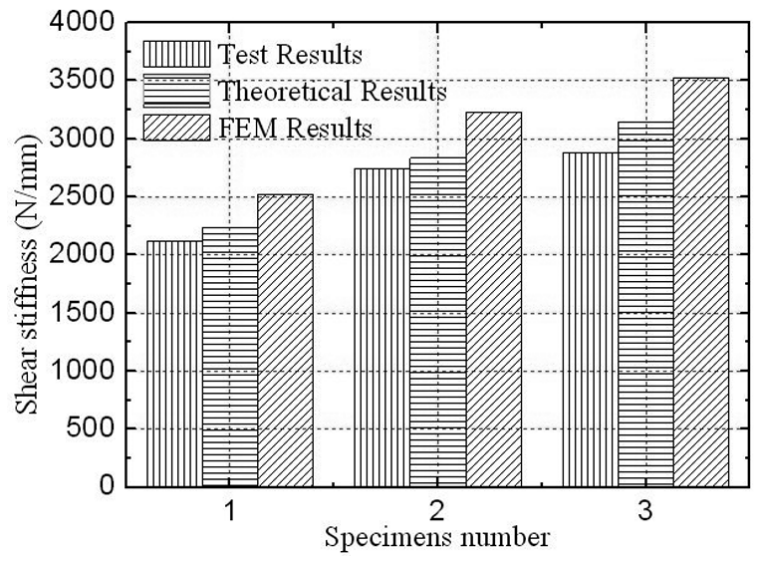

Fig. 18. Comparison of compression stiffness results of different methods.

\subsection{Stress analysis of FRPEIS}

The stress distribution in the rectangular layer of the rubber bonded to the FRP reinforcement of specimen No. 2 when it is subjected to $40 \mathrm{MPa}$ pressure load is shown in Fig. 16. Figures 16(a) and (b) are the stress distribution results of FEM and theoretical equation derived by Tsai [8], respectively. The differences between the two methods are small; hence they can verify each other. Figure 16 indicates that the center of the rubber layer has the highest pressure, which is nearly two times of the vertical load applied to the specimen.

Figure 17 presents the stress distribution in FRP reinforcement of specimen No. 2 when it is subjected to $70 \mathrm{MPa}$ pressure load. It shows that the center of the reinforcement has the highest stress, which is about $530 \mathrm{MPa}$. Therefore, if rubber and reinforcement are bonded perfectly in the specimen, the center of the reinforcement will break out first when it reaches ultimate vertical load. This is verified by the fact that the reinforcements of the specimens are mostly broken from the center at the end of vertical bearing capacity tests.

\subsection{Stiffness analysis of FRPEIs}

The comparison of compression modulus results of theoretical analyses, FEM analyses, and experiments is shown in Fig. 18. The theoretical expression of compression modulus is derived by Tsai [8] and the test results are from Section 3.1. It's shown that the difference between the results of theoretical and FEM analyses is less than 20\%. The results of theoretical and FEM analyses are larger than the test results, and the maximum error is almost $40 \%$. 


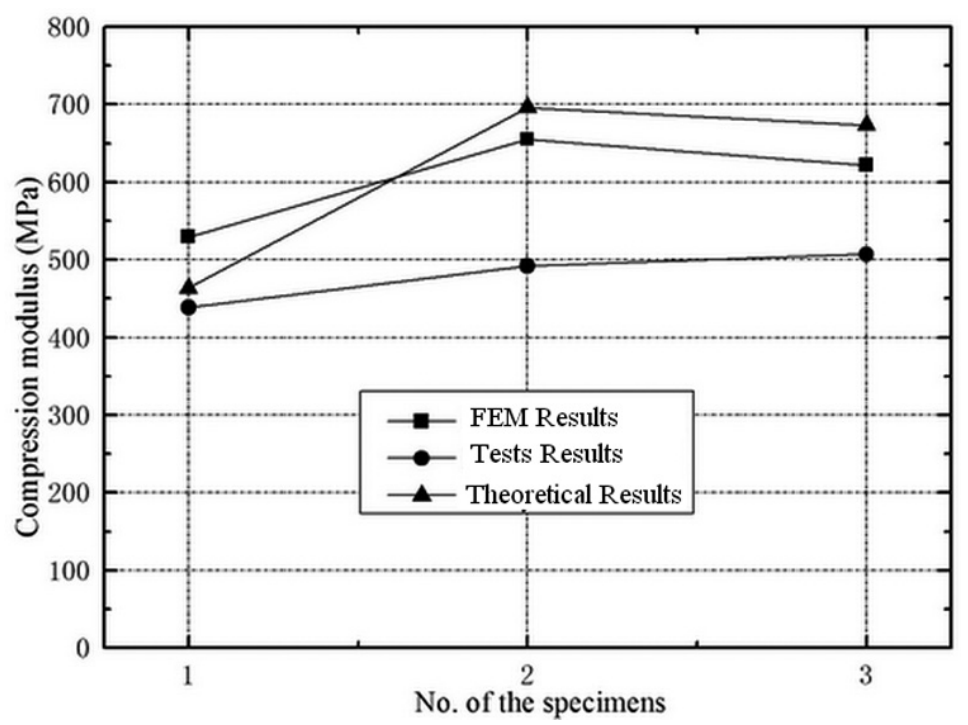

Fig. 19. Comparison of shear stiffness results of different methods.

The errors may be caused by the defects in the production process, the measure errors in the test process and the ideal incompressibility assumption of rubber material in FEM and theoretical analyses. Where, production defects may exist in the rubber, the fibre fabric and epoxy resin, and they will decrease the stiffness, but their influences are stochastic. The influences of measure error are also stochastic and the assumptions of incompressibility will increase the compression stiffness results of FEM and theoretical analyses definitely.

The shear stiffness results of the three specimens are studied in detail with experiments, FEM and theoretical analyses. The shear stiffness results of theoretical analyses can be calculated as follows:

$$
K_{s}=\frac{G A}{\sum t_{r}}
$$

where $G$ is the shear modulus of the rubber for the isolator and material tests have been conducted to determine the parameters in the test specimens, $A$ is the planar area of the isolator and $\sum t_{r}$ is the total thickness of the rubber in the isolator.

The results of different methods are shown in Fig. 19, from which it can be learnt that the theoretical results are larger than the experimental results, and the FEM results are the largest among these three methods. The lower results with experimental method may be caused by the inevitable defects in rubber material and in the manufacturing process. Moreover, the theoretical expression of the shear stiffness of multilayer elastomeric isolator is derived from the assumption that the reinforcement in the isolator is rigid (have no shear strain), but the fibre reinforcement can have small shear strain, so the theoretical results can be a little larger. Since the rubber is assumed to be an ideal hyperelastic and incompressible material in FEM analyses, the FEM results (calculated from the loading condition with the same shear strain to the experimental method) are a little larger than those of the other two methods. If the theoretical method is used to design FRPEIs, a reduction factor for stiffness calculation should be used to account for the defects in the production process.

\section{Conclusions}

A new kind of bridge seismic isolator named fibre-reinforced plate elastomeric isolator (FRPEI) is developed in this paper. To investigate the mechanical performance of FRPEI, specimens with glass fibre-reinforced plate were manufactured. Vertical tests are carried out to evaluate the compression stiffness results and vertical bearing capacities of FRPEIs, while horizontal tests are conducted to study the shear stiffness and hysteretic characteristics. 
The results show that FRPEI has similar stiffness to that of laminated rubber bearing reinforced with steel plates, and the vertical bearing capacity can meet the requirement of service load. Meanwhile, compared to the laminated rubber bearing reinforced with steel plates, FRPEI has better energy dissipation capacity since it has hysteretic damping, which indicates that FRPEI has advantages for seismic isolation design of bridge structures.

Finite element method is applied to simulate the test processes. Firstly, the deformation assumption in theoretical analyses is verified by fitting the FEM results with quadratic polynomial. Then the stress distribution in FRP reinforcement is analyzed to study the failure mode of the bearing under ultimate vertical load. It shows that the failure of the isolator is possibly due to the rupture at the center of the reinforcement. Comparisons of the compression and shear stiffness results of different methods are carried out and test results are always less than the other two results, which probably because of the defects in the production process, the measure errors in the test process and the ideal incompressibility assumption of rubber material in FEM and theoretical analyses.

\section{Acknowledgments}

The authors gratefully acknowledge the financial support provided by the National Natural Science Foundation of China (No. 51278372), National Key Technology R\&D Program (No. 2009BAG15B01), the Ministry of Science and Technology of China, Grant No. SLDRCE 08-B-04, the Fundamental Research Funds for the Central Universities and Kwang-Hua Fund for College of Civil Engineering, Tongji University. The opinions and conclusions presented in this paper are those of the authors and do not necessarily reflect the views of the sponsoring organizations.

\section{References}

[1] N. Mostaghel, Resilient-friction base isolator, Report No. UTEC 84/97, Department of Civil Engineering, University of Utah, Salt Lake City, 1984.

[2] M.C. Constantinou and M.D. Symans, Experimental and analytical investigation of seismic response of structures with supplemental fluid viscous dampers, Report No. NCEER-92-0032, National Center for Earthquake Engineering Research, State University of New York at Buffalo, Buffalo, 1992

[3] C.J. Derham, J.M. Kelly and A.G. Thomas, Nonlinear natural rubber bearings for seismic isolation, Nuclear Engineering Design 84(3) (1985), 417-428.

[4] W.H. Robinson, Lead-rubber hysteretic bearing suitable for protecting structures during earthquakes, Earthquake Engineering \& Structural Dynamics 10 (1982), 593-604.

[5] J.M. Kelly, Analysis of fibre-reinforced elastomeric isolators, J Seismol Earthq Eng 2(1) (1999), 19-34.

[6] J.M. Kelly and S.M. Takhirov, Analytical and experimental study of fibre-reinforced elastomeric isolators, PEER Rep No 2001/11, Berkeley (CA, USA): Pacific Earthquake Engineering Research Center, Univ of California, 2001.

[7] J.M. Kelly and S.M. Takhirov, Analytical and experimental study of fiber-reinforced strip isolators, PEER Rep No 2002/11, Berkeley (CA, USA): Pacific Earthquake Engineering Research Center, Univ of California, 2002.

[8] H.C. Tsai and J.M. Kelly, Stiffness analysis of fibre-reinforced elastomeric isolators, PEER Rep No 2001/05, Berkeley (CA, USA): Pacific Earthquake Engineering Research Center, Univ of California, 2001.

[9] H.C. Tsai and J.M. Kelly, Stiffness analysis of fiber-reinforced rectangular seismic isolators, J Eng Mech 128(4) (2002), 462-470.

[10] H.C. Tsai and J.M. Kelly, Bending stiffness of fiber-reinforced circular seismic isolators, J Eng Mech 128(11) (2002), 1150-1157.

[11] H.C. Tsai, Compression stiffness of infinite-strip bearings of laminated elastic material interleaving with flexible reinforcements, Int $J$ Solids Struct 41(24-25) (2004), 6647-6660.

[12] H.C. Tsai and J.M. Kelly, Buckling load of seismic isolators affected by flexibility of reinforcement, Int J Solids Struct 42(1) (2005), 255-269.

[13] B.-Y. Moon, G.-J. Kang, B.-S. Kang and J.M. Kelly, Design and manufacturing of fibre reinforced elastomeric isolator for seismic isolation, J Mater Process Technol 130/131 (2002), 145-150.

[14] B.-Y. Moon, G.-J. Kang, B.-S. Kang, G.-S. Kim and J.M. Kelly, Mechanical properties of seismic isolation system with fibre-reinforced bearing of strip type, Int Appl Mech 39(10) (2003), 1231-1239.

[15] B.-S. Kang, G.-J. Kang and B.-Y. Moon, Hole and lead plug effect on fibre reinforced elastomeric isolator for seismic isolation, $J$ Mater Process Technol 140 (2003), 592-597.

[16] A. Mordini and A. Strauss, An innovative earthquake isolation system using fibre reinforced rubber bearings, Eng Struct 30 (2008), 2739-2751.

[17] G.-D. Ashkezari, A.-A. Aghakouchak and M. Kokabi, Design, manufacturing and evaluation of the performance of steel like fibre reinforced elastomeric seismic isolators, J Mater Process Technol 197 (2008), 140-150.

[18] Ministry of Communications of PRC JT/T4-2004, Plate type elastomeric pad bearings for highway bridges (in Chinese), 2004.

[19] M.J.N. Priestley, F. Seible and G.M. Calvi, Seismic design and retrofit of bridges, Jonhn Wiley \& Sons, Inc., 1996. 

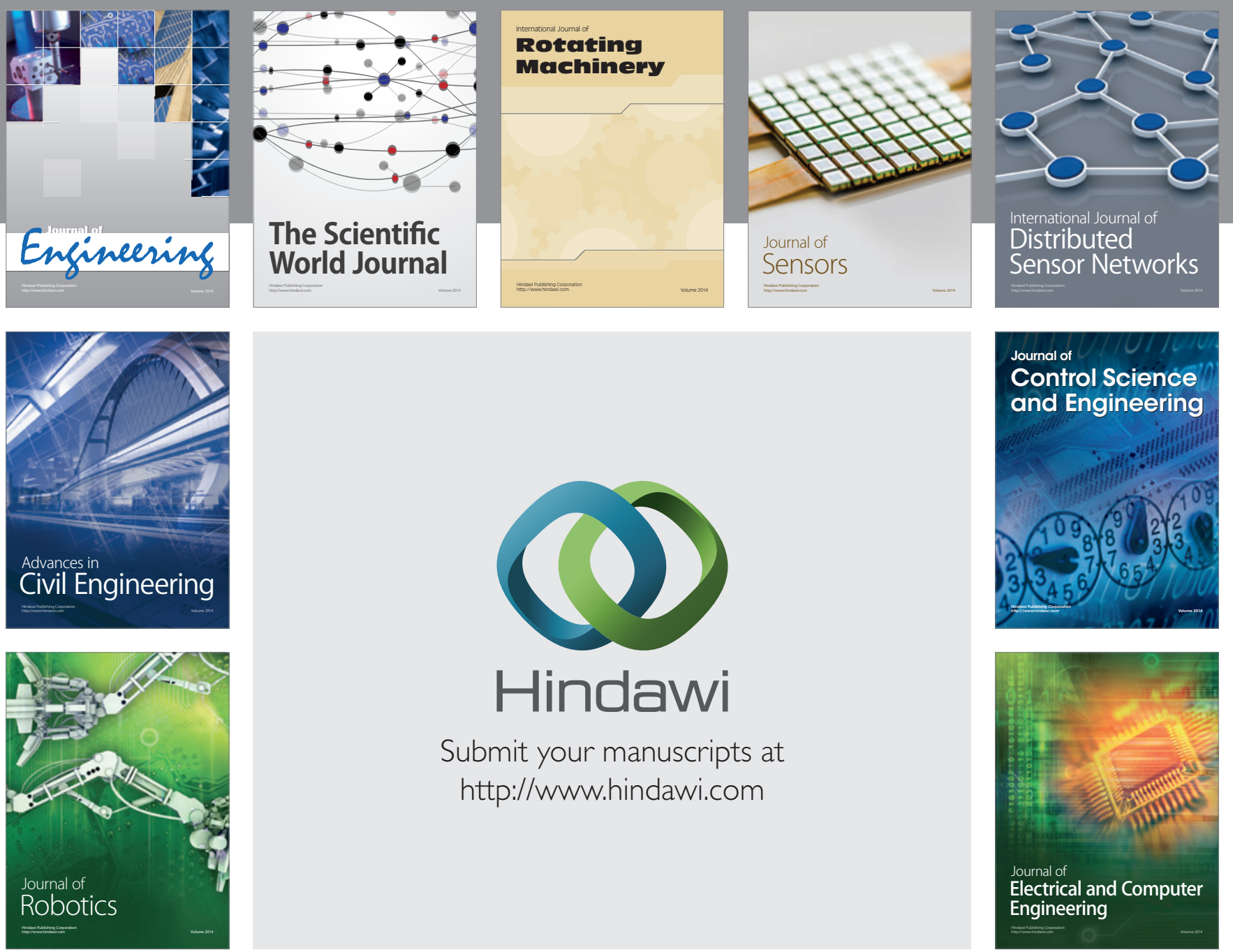

Submit your manuscripts at

http://www.hindawi.com
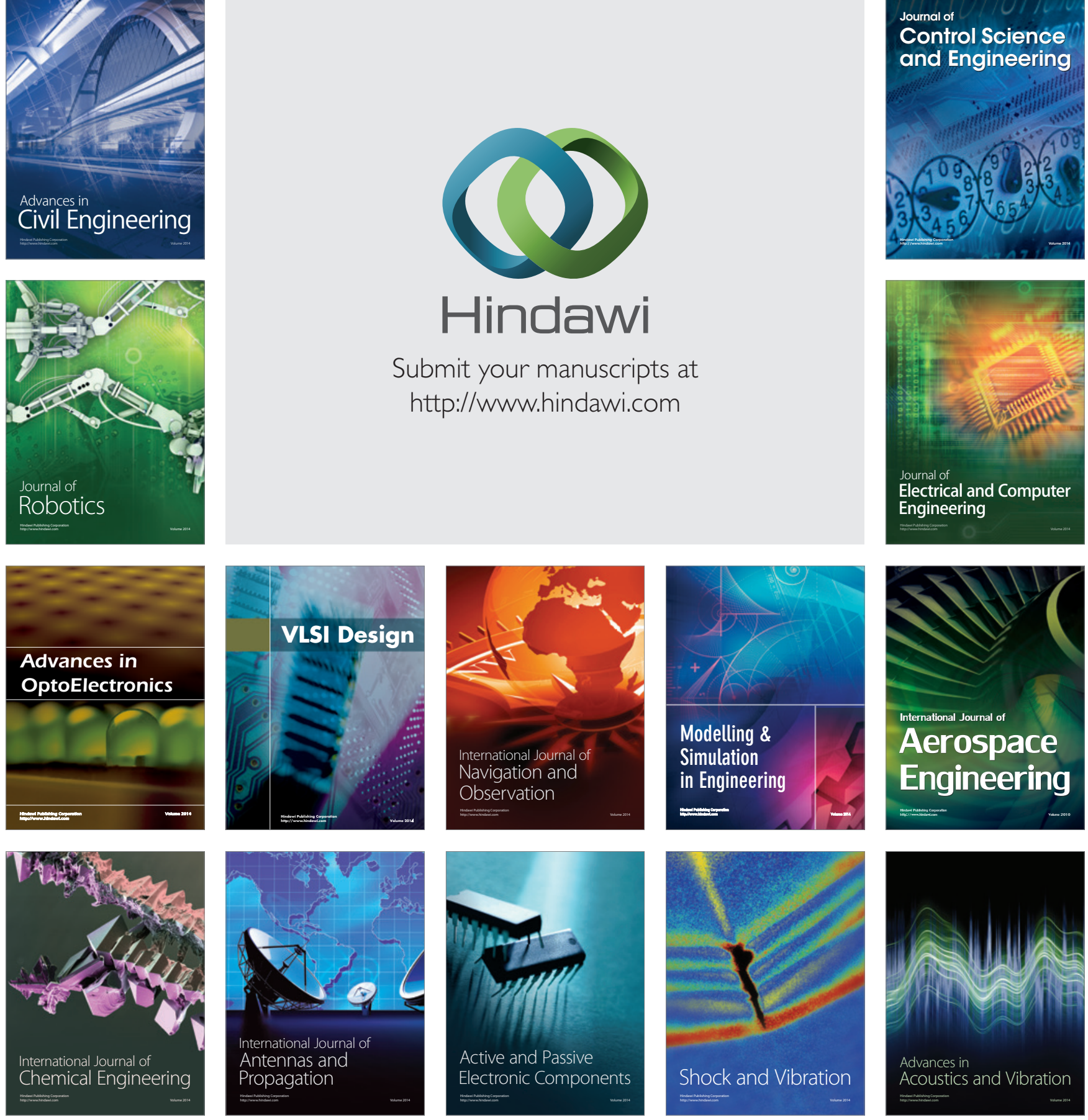